\title{
Information and Risk Modification Trial (INFORM): design of a randomised controlled trial of communicating different types of information about coronary heart disease risk, alongside lifestyle advice, to achieve change in health-related behaviour
}

Barbora Silarova', Joanne Lucas², Adam S. Butterworth²,3, Emanuele Di Angelantonio 2,3, Christine Girling4, Kathryn Lawrence ${ }^{4}$, Stuart Mackintosh ${ }^{2}$, Carmel Moore ${ }^{2,3}$, Rupert A. Payne ${ }^{5}$, Stephen J. Sharp ${ }^{1}$, Guy Shefer ${ }^{1}$, Zoe Tolkien ${ }^{2,3}$, Juliet Usher-Smith ${ }^{6}$, Matthew Walker ${ }^{2,3}$, John Danesh ${ }^{2,3}$ and Simon Griffin ${ }^{1,6^{*}}$ (D)

\begin{abstract}
Background: Cardiovascular disease (CVD) remains the leading cause of death globally. Primary prevention of CVD requires cost-effective strategies to identify individuals at high risk in order to help target preventive interventions. An integral part of this approach is the use of CVD risk scores. Limitations in previous studies have prevented reliable inference about the potential advantages and the potential harms of using CVD risk scores as part of preventive strategies. We aim to evaluate short-term effects of providing different types of information about coronary heart disease (CHD) risk, alongside lifestyle advice, on health-related behaviours.
\end{abstract}

Methods/Design: In a parallel-group, open randomised trial, we are allocating 932 male and female blood donors with no previous history of CVD aged 40-84 years in England to either no intervention (control group) or to one of three active intervention groups: i) lifestyle advice only; ii) lifestyle advice plus information on estimated 10-year CHD risk based on phenotypic characteristics; and iii) lifestyle advice plus information on estimated 10-year CHD risk based on phenotypic and genetic characteristics. The primary outcome is change in objectively measured physical activity. Secondary outcomes include: objectively measured dietary behaviours; cardiovascular risk factors; current medication and healthcare usage; perceived risk; cognitive evaluation of provision of CHD risk scores; and psychological outcomes. The follow-up assessment takes place 12 weeks after randomisation. The experiences, attitudes and concerns of a subset of participants will be also studied using individual interviews and focus groups.

Discussion: The INFORM study has been designed to provide robust findings about the short-term effects of providing different types of information on estimated 10-year CHD risk and lifestyle advice on health-related behaviours. (Continued on next page)

\footnotetext{
* Correspondence: sjg49@medschl.cam.ac.uk

'MRC Epidemiology Unit, University of Cambridge, Institute of Metabolic Science, Cambridge CB2 OQQ, UK

${ }^{6}$ The Primary Care Unit, Department of Public Health and Primary Care, University of Cambridge, Strangeways Research Laboratory, 2 Wort's Causeway, Cambridge CB1 8RN, UK

Full list of author information is available at the end of the article
} 
(Continued from previous page)

Trial registration: Current Controlled Trials ISRCTN17721237. Registered 12 January 2015.

Keywords: Behaviour, Cardiovascular diseases, Communication, Genetic, Phenotypic, Physical activity, Protocol, Randomised controlled trial, Risk

\section{Background}

Cardiovascular disease (CVD) remains the leading cause of death globally [1], with an estimated 17 million deaths from CVD in 2011, or about 3 in every 10 deaths [2]. Of CVD deaths, 7 million deaths are due to coronary heart disease (CHD) and 6.2 million due to stroke [3]. The annual number of people who die from CVD is predicted to reach 23.3 million by 2030 and CVD is projected to remain the single leading cause of death [4].

Primary prevention strategies often involve CVD risk scores [5-9] to identify individuals at high estimated risk of CVD in order to target preventive interventions. Risk scores, which are recommended for use by national guidelines in most countries [10, 11], are algorithms that calculate an individual's risk of CVD by combining information on several different risk factors. In the United Kingdom, CVD risk assessment tools are implemented in general practitioner (GP) computer systems [12]. The National Health Service (NHS) has initiated a programme of CVD risk reduction ("NHS Health Check") which includes assessment of CVD risk for all those aged 40-74 years without pre-existing CVD and related disorders, although the effectiveness of this approach remains uncertain [13].

There are several risk factors that increase the risk of CVD including both non-modifiable factors such as family history, ethnic origin and gender and modifiable risk factors such as smoking, unhealthy diet, obesity and diabetes $[14,15]$. The UK National Institute for Health and Care Excellence (NICE) [16] and the Joint British Societies for the prevention of CVD [10] recommend primary prevention of CVD through a focus on interventions that may change the behaviour of individuals, such as smoking cessation, reducing alcohol consumption, and increasing physical activity [17]. The provision of CVD risk information could afford the opportunity to improve CVD risk perception among individuals and so motivate a change in health-related behaviours. There is evidence to suggest that the provision of phenotypic CVD risk information improves people's accuracy of perceived risk. Systematic reviews have, however, reported that provision of risk information needs to be accompanied by other tools in order to improve health-related behaviours and other relevant clinical outcomes $[18,19]$.
Studies included in previous reviews have been limited by the use of self-reported (rather than objective) measures of diet and physical activity.

Recent identification of several genetic risk factors for CVD has suggested the possibility of supplementing traditional CVD risk scores with genetic information to help motivate behaviour change and improve clinical outcomes [20, 21]. Theoretical models of health-related behaviour change, such as the Health Belief Model [22], would predict a positive impact of using such an approach. Direct-to-consumer genetics companies have claimed that providing people with such information could encourage healthy lifestyle choices. Alternatively, however, provision of such information could induce a defeatist response, leading to unhealthy lifestyle choices. A review by Marteau et al. [23] concluded that provision of genetic risk information generally has little or no effect on behaviour, although it may have a small effect on intentions to change behaviour. However, previous studies of the impact of provision of genetic risk information have been underpowered and lacked objective measures of behaviour. Hence, the potential benefits and the potential harms of adding, and communicating, genetic CVD risk information to phenotypic CVD scores are unknown.

Using both quantitative and qualitative methods, we aim to assess the short-term effect of providing different types of information about CHD risk, alongside lifestyle advice, on health-related behaviours.

\section{Objectives \\ Primary objective}

The primary objective of the INFORM study is to evaluate the effect of providing different types of information about estimated 10-year CHD risk, alongside lifestyle advice on health-related behaviours over three months.

\section{Secondary objectives}

The secondary objectives are to evaluate the effect of providing different types of information about $\mathrm{CHD}$ risk, alongside lifestyle advice on objectively measured dietary behaviour (key secondary objective); cardiovascular risk factors; current medication and healthcare usage; perceived risk; cognitive evaluation of provision of CHD risk scores; and psychological outcomes. 


\section{Objectives of the qualitative study}

The study also aims to provide rich and detailed information which may explain, clarify, support or modify the quantitative findings.

\section{Methods/Design \\ Study design}

The design of the trial and flow of participants are shown in Fig. 1. In a parallel-group, open randomised trial, we are allocating 932 male and female blood donors with no previous history of CVD aged 40-84 years in England to either no intervention (control group) or to one of three active intervention groups: i) lifestyle advice only ii) lifestyle advice plus information on estimated 10-year CHD risk based on phenotypic characteristics; and iii) lifestyle advice plus information on estimated 10-year CHD risk based on phenotypic and genetic characteristics. Lifestyle advice consists of three sessions of interactive, tailored web-based information. The qualitative element of the study consists of individual interviews and focus groups with trial participants at different time points throughout the trial.

\section{Population}

Male and female blood donors aged 40-84 years with no previous history of CVD.

\section{Setting}

The trial is administered at the University of Cambridge. Participants live in England.

\section{Recruitment}

Participants are a subset of those recruited into the INTERVAL study, an ongoing randomised, multisite trial that has recruited approximately 50,000 blood donors from all 25 permanent donor centres of NHS Blood and Transplant (NHSBT) throughout England. The INTERVAL study aims to determine whether donation intervals can be safely and acceptably decreased to optimise blood supply while maintaining the health of donors [24]. Blood donors are potentially eligible to participate in the INTERVAL study if they are aged 18 years or older, fulfil all normal criteria for blood donation [25] and are willing to donate at one of the permanent NHSBT donation centres over a period of two years. Participants are excluded if they do not have internet access and/or are not

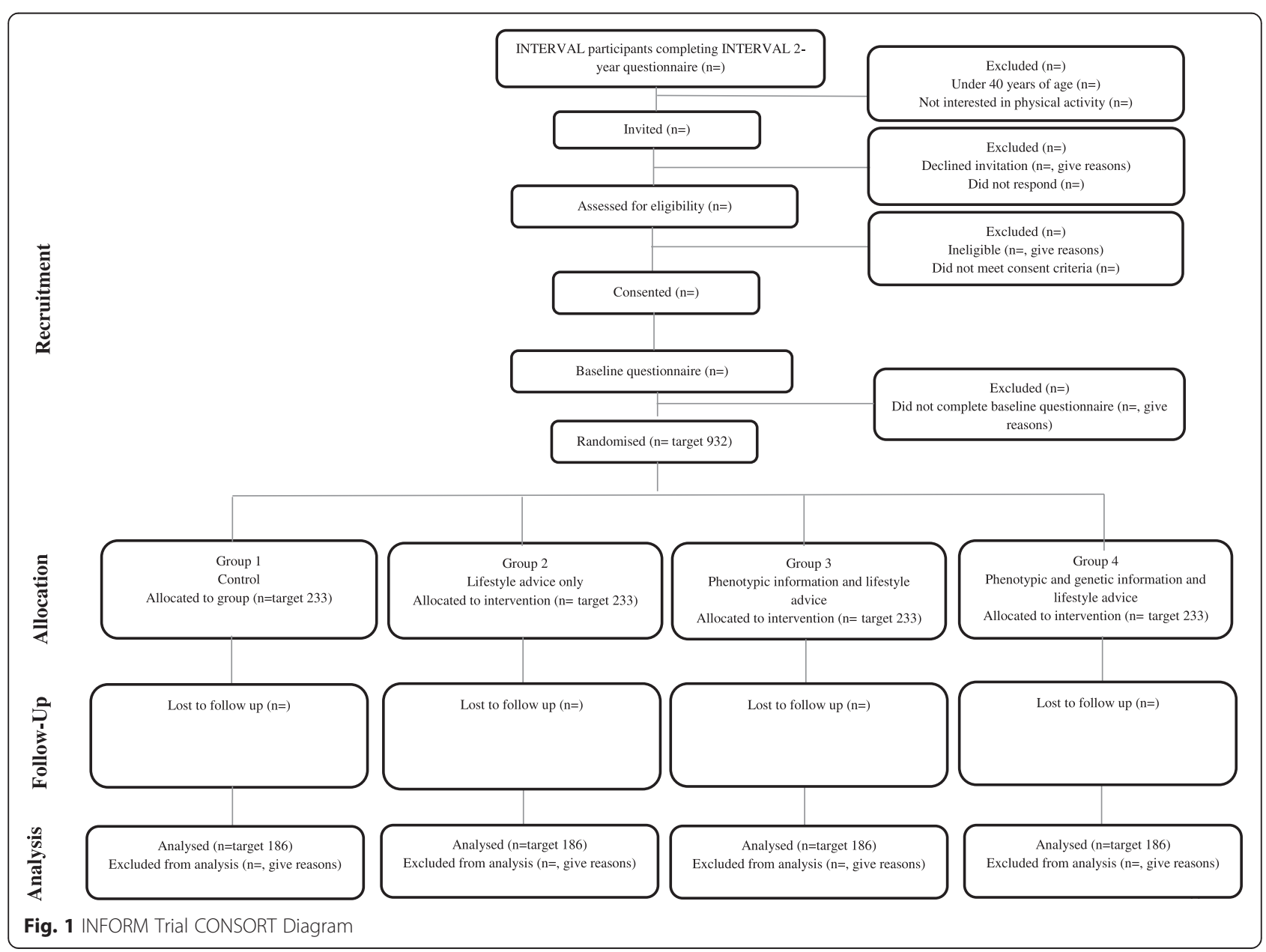


willing to provide an email address for trial correspondence, since the trial mainly collects data via remote and web-based methods [24]. INTERVAL participants provide data (via online questionnaires) and blood samples at the time of joining the study and after two years. DNA has been extracted from baseline blood samples for genome-wide genotyping using the Affymetrix "UK Biobank" Axiom genotyping array. In addition, lipid profiles of all participants are being assessed via NMRmetabolomic assay. At their 2-year anniversary of joining INTERVAL, a subset of participants, who are willing and selected to do so, wear a physical activity monitoring device for 7 days (Axivity AX3 3-Axis Logging Accelerometer ${ }^{\circ}$, Axivity, York, UK).

INTERVAL participants are invited to take part in the INFORM study if they have completed their INTERVAL 2-year follow-up questionnaire and have indicated an interest in wearing a physical activity monitor. Furthermore, they have to be aged 40-84 years (inclusive); willing to provide a blood sample; have sufficient data available to the study team for calculation of phenotypic and genetic risk estimates for CHD (described in detail below); agree to allow trial staff to contact their GP to notify them of trial participation and study test results; have internet access and are willing to provide an email address for study correspondence; and have a good understanding of the English language, both written and oral (study materials are not tailored to support nonEnglish language speakers). Potential participants are excluded if they have prior history of CVD (e.g. heart attack, angina, peripheral arterial disease or stroke, surgical or percutaneous coronary revascularisation procedure); a medical condition or disability that means the participant cannot engage in physical activity; known pregnancy at time of recruitment; are unable to provide informed consent; and are currently participating in another interventional clinical trial in cardiovascular risk or lifestyle modification (e.g. diet, physical activity or smoking cessation).

Between March 2015 and June 2015, electronic invitations approved by the local ethics committee have been sent to participants who meet the invitation criteria. The invitation email message consists of a brief summary and a personalised weblink to the INFORM screening and consent questionnaire. It also contains a weblink to the study website [26], where participant information is available.

We aim to involve a subset of 30-35 participants in the INFORM trial to take part in qualitative interviews and a further subset of 16-36 participants to take part in 2-3 focus groups. The exact number of the interviewees and focus groups is dependent on saturation of the data and the willingness of enough participants from the same area to take part in focus groups. Invitations to take part in the qualitative interviews and focus groups are sent to a purposive sample selected from participants who have given their consent to be approached about the qualitative study when completing the online consent form to take part in the trial. The invitation email message for the qualitative study consists of an invitation and participant information leaflet.

\section{Consent}

Potential participants are providing implied consent for the INFORM Screening questionnaire by completing the questionnaire and those who are eligible progress to online informed consent for study entry. As part of the informed consent participants provide consent to provide a blood sample. When participants visit their general practice to have their blood sample taken, practice staff will take verbal consent for the procedure at the time of the visit and this will be recorded in the patient notes. The qualitative interview and focus group participants will be asked to sign a paper consent form before the start of the interview or focus group.

\section{Baseline assessment}

Baseline information is collected prior to randomisation. Responders who are eligible to take part in the INFORM study and provide informed consent are sent a welcome email message with an individually tagged URL link to the baseline online questionnaire (available from the authors on request). Participants are requested to complete this questionnaire within 7 days and receive reminder email messages at day 7 and day 14 . A reminder telephone call will be made on day 16 if the questionnaire still has not been completed. The measures assessed in the baseline questionnaire are combined with several measures taken during the participants' INTERVAL study assessment in order to characterise the study population and to calculate individual's phenotypic and genetic risk scores.

For consenting participants in INFORM who took part in physical activity monitoring in the INTERVAL study, physical activity data will be shared between studies and used as a baseline measurement for the INFORM study. Participants who did not take part in physical activity monitoring during the INTERVAL study are required to undertake baseline physical activity monitoring over 7 days. The study team sends participants a watch-like device (Axivity AX3 3-Axis Logging Accelerometer ${ }^{\circ}$ ) to measure physical activity over a period of 7 days. The device is sent together with instructions and a list of frequently asked questions and responses to the questions. A letter detailing the "switch on" and "switch off" date (pre-set by the study team before posting) is included with the device with instructions for return to the study team in the freepost envelope provided. Participants are 
reminded by email to return their monitor 6 days after the "switch off" date. If the monitor is not returned after the email message is received, the helpdesk will attempt to contact the participant by telephone or email. If a response is still not received, a written request for the return of the monitor will be sent after 5 weeks. If the monitor is still not returned after 6 weeks, the participant is randomised.

\section{Randomisation, allocation concealment and blinding}

Randomisation of the INFORM participants is undertaken centrally at the trial coordinating centre at the Department of Public Health and Primary Care, University of Cambridge. The study involves individual-level randomisation using a computer program built into the study database. This programme is designed and implemented by the data manager. Randomisation is stratified by age ( $\leq$ or $>60$ years) and sex in order to balance baseline phenotypic risk across study groups. Given the nature of the trial, it is not possible to blind participants to which intervention they receive. However, an independent data manager (not involved in designing the study) oversees the randomisation and data collection process. Additionally, the researchers assessing the trial outcomes will remain blinded to the allocation of interventions. As the participants for the qualitative part of the study are purposively selected, the qualitative researchers have access to the information collected during baseline assessment and treat it as confidential.

\section{Interventions}

The study arms are summarised in Table 1. All participants will receive any information omitted from their group allocation at the end of the follow-up period (this includes the control group). The intervention in the INFORM study consists of three components provided to groups as described in Table 1: phenotypic CHD risk score; genetic CHD risk score; and lifestyle advice.

\section{Phenotypic CHD risk score}

The phenotypic CHD risk score consists of three pieces of information (Additional file 1A for example) as adapted from a study by Persell et al. [27]. First, we provide participants with information on their absolute risk of having CHD in the next 10 years, both as a percentage and as a natural frequency. We used the same set of traditional CVD risk factors (age, sex, smoking status, blood pressure, diabetes mellitus and total cholesterol and high-density lipoprotein - HDL - cholesterol) as used in many existing CVD risk scores [28] to calculate the absolute risk of having either fatal or nonfatal CHD events during the next 10 years. However, instead of objectively measured systolic blood pressure (as used in existing CVD risk scores), we use self-reported information on prescribed antihypertensive medication as a proxy for blood pressure (since it is not logistically feasible to collect objectively measured blood pressure in this study). We therefore refined an existing validated phenotypic CVD risk score [29] to take account of this change in variable. In line with D'Agostino et al. [29], we used sex-specific Cox proportional-hazards regressions to relate risk factors to the incidence of first CHD event. Correlates included in the Cox models were age, total cholesterol (mmol/l), HDL cholesterol ( $\mathrm{mmol} / \mathrm{l})$, antihypertensive medication (self-reported yes/no), current smoking (self-reported yes/no) and diabetes mellitus (self-reported yes/no). All the continuous variables were log-transformed. From these models, we estimated mathematical CHD functions to predict 10-year risk of $\mathrm{CHD}$, using the general formula as published by $\mathrm{D}^{\prime}$ Agostino et al. [29].

Second, the absolute risk of having CHD in the next 10 years is accompanied by "Heart Age". An individual's heart age is the chronological age of someone with the same absolute risk of CHD but with healthy risk factors. This means that individuals with elevated CHD risk factors will have a higher heart age than their chronological age. The definition of "normal" is based on the following profile: not a current smoker, does not have diabetes, not using antihypertensive medication, total serum cholesterol $=4.6548 \mathrm{mmol} / \mathrm{l}$ and HDL cholesterol $=1.1637 \mathrm{mmol} /$ 1 [30]. The "Heart Age" was derived from the same general formula as used for the absolute risk score, rearranged such that age is unknown, other risk factors are normal (as defined above) and absolute risk is that of the participant in question.

Finally, we provide participants with a comparative risk estimate to encourage changes in health-related behaviours. An individual's comparative risk is the risk of someone who is the same age and sex and has healthy lifestyle-related factors that are associated with $\mathrm{CHD}$ risk $[31,32]$. Healthy lifestyle-related factors were defined as

Table 1 Study Arms

\begin{tabular}{|c|c|c|c|c|}
\hline Group & Type & Lifestyle advice & Phenotypic risk score & Genetic risk score \\
\hline 1 & Control & No & No & No \\
\hline 2 & Intervention & Yes & No & No \\
\hline 3 & Intervention & Yes & Yes & No \\
\hline 4 & Intervention & Yes & Yes & Yes \\
\hline
\end{tabular}


follows: a) current non-smokers (i.e. never smoked and former smokers) [33]; b) moderate levels of alcohol consumption (one or more units a week but not more than fourteen units a week; 1 unit = 8 grams) [33]; c) consumption of fruit and vegetables (more than 400 grams) [34]; d) consumption of fish (a portion size of 140 grams cooked weigh (20 grams/day) [35]; e) consumption of red meat ( $\leq 6$ portions a week, equivalent to $\leq 500$ grams of cooked weight or 71 grams per day [36]; f) physical activity (not inactive, at least half an hour of leisure-time activity a day) [33]; g) body mass index (BMI) $<25 \mathrm{~kg} / \mathrm{m}^{2}$.

All three pieces of information (absolute risk, "Heart Age" and comparative risk) provided as part of the phenotypic CHD risk score were modelled using the data from the EPIC-Norfolk study [37]. This means that we ran Cox proportional-hazards regressions as described above in EPIC-Norfolk to get the coefficients, but each INFORM participant's values are plugged in to these equations to get their 10-year risk (Additional file 1B for example). As the outcome for modelling, we have chosen CHD instead of CVD, since the majority of genomic loci known to relate to CVD are associated with CHD (rather than stroke) and the study we used to estimate the coefficient for our genetic risk score (EPIC-CVD) only has CHD endpoints available currently. Given the fact that CHD is the most common type of CVD the results of INFORM study should be also applicable for CVD risk communication.

\section{Genetic CHD risk score}

As with the phenotypic CHD risk score, the genetic CHD risk information consists of three pieces of information (Additional file 1C for example). First, we provide participants with the information on their absolute risk of having CHD in the next 10 years. Second, information on "Heart Age" is provided. Lastly, we provide participants with a comparative risk estimate.

At the time of study design, there were 49 known genomic loci robustly associated with risk of CHD [38]. We selected lead variants at 46 of these regions that were included on the Affymetrix "UK BioBank" Axiom genotyping array being used on INTERVAL participants, as well as a customised version of the Illumina "Exomechip" array that has been run in the EPIC-CVD study [39], which was used to derive the risk estimates. EPIC-CVD is a case-cohort study comprising of $\sim 10,000$ incident CHD cases and a similar number of randomly selected comparators. Participants who have been genotyped in the INTERVAL study have a weighted genetic risk score (GRS) estimated for them based on their genotypes at each of the 46 relevant variants (Additional file 1D). This means that for each individual, the number of CHD risk alleles $(0 / 1 / 2)$ carried at each single nucleotide polymorphism
(SNP) was weighted by the strength (log of the odds ratio) of that SNP's association with risk of disease (to account for the fact that not all variants have equal strengths of association) and then summed across SNPs. Weights used were drawn from the replication or combined estimates of association from the studies that originally discovered the variant associations [38, 40-42] . For estimation of the association between the GRS and incident CHD outcomes, the GRS was natural log-transformed (lnGRS). In line with the phenotypic risk score, we estimated mathematical CHD functions to predict 10-year risk of CHD using a similar general formula. For the absolute genetic risk score, this formula was plugged with the same baseline cumulative hazard as the absolute phenotypic risk score, individual $\ln$ GRS and the mean lnGRS. For the purpose of "Heart Age", normal lnGRS was defined as the sex-specific 10th centile ( $<1.043$ for males, $<1.045$ for females). For the purpose of comparative risk estimate, healthy lnGRS was defined as bottom $10 \%$ of $\operatorname{lnGRS}$ (Additional file $1 \mathrm{E}$ for example).

\section{Format of phenotypic and genetic risk}

Both CHD risk scores are presented in the same format to ensure that the only experimental condition is the addition of information on the risk of CHD based on genetic variants (and not the different formats of presenting the CHD risk information). In designing the CHD risk scores we have taken into account the evidence regarding the most effective methods for communicating CHD risk estimates [30, 43-46]. Based on this evidence, INFORM participants are provided with: i) a graphical format in the shape of a thermometer using colours to distinguish risk (red, yellow, green) [47], ii) "Heart Age" tool and iii) comparative risk information [45, 47] (Additional file $1 \mathrm{~A}$ and $1 \mathrm{C}$ for example). In addition to the CHD risk estimates, we also provide participants with an explanation of the factors included in the calculation of their CHD risk scores, how to interpret the thermometer and who to contact in case of any questions, as well as a section with the answers to frequently asked questions (adapted from a randomised trial of personal genomics for preventive cardiology [48]; and from a study by Persell et al. [27]) in line with previous evidence that participants expressed a need to understand the numbers and values presented to them [49].

\section{Lifestyle advice}

For the purpose of the INFORM study, we are using a web-based lifestyle intervention for CHD prevention based on an intervention that was originally developed for the Heart to Health study [50]. The lifestyle intervention consists of three sessions of interactive, tailored information on the web (up to three hours of interventional 
contact). In previous studies, offering patients a riskreducing strategy was associated with increased adherence and reduced CHD risk [51]. Therefore, a library of over 100 web pages providing advice on physical activity, diet, smoking and medication are tailored according to participants' selection of risk-reducing strategies (the web pages are available from the authors on request). Each session may last up to $60 \mathrm{~min}$ in duration depending on participants' individual pace with sessions delivered at monthly intervals. The first session begins with content-specific education on diet, physical activity and smoking cessation. This session also includes tips on how to overcome selfidentified barriers to risk reduction and the creation of steps toward self-identified actionable goals. Sessions 2 and 3 include similar content; a participant begins by reviewing their progress toward goals, continues with education and tips to overcome barriers and finishes with identification of new goals [50].

\section{Follow-up assessment}

Follow-up assessments take place at 12 weeks after randomisation and include an online questionnaire, physical activity monitoring and blood sample collection. Not all of the measures in the baseline questionnaire are repeated at follow up (Table 2). The follow-up questionnaire (available from the authors on request) should take no more than $20 \mathrm{~min}$ to complete. Participants are requested to complete this follow-up questionnaire and will receive two reminder emails at day 7 and day 14 after sending the follow-up questionnaire if it has not been completed. If we do not hear from participants by day 24 , we will mark the data as missing.

\section{Follow-up physical activity monitoring}

All participants are requested to complete physical activity monitoring for 7 days at the 12-week time point following the same procedure as at baseline.

\section{Blood sample}

All participants are asked to provide a blood sample, which is taken at their general practice. The blood sample is used to provide information on participants' lipid panel (total, HDL and low-density lipoprotein - LDL cholesterol; triglycerides), carotenoid levels and fructosamine level. The study coordination team provides a blood sample kit (Royal Mail Safebox ${ }^{\mathrm{TM}}$ ) which is sent to participants along with the follow-up physical activity monitor. The blood sample kit includes blood collection tubes with barcoded, anonymised participant study number, instructions for general practice clinicians, general practice invoicing instructions and appropriate packaging addressed to the receiving central laboratory with pre-paid postage. A staff member at the participant's general practice takes verbal consent for the procedure at the time it is taken, writes the date and time that the sample was taken on the blood collection tube, packs the sample, and enters it into the postal delivery chain alongside regular practice post. Samples are transported by Royal Mail first class mail to a central laboratory (UK BioCentre, Stockport, Cheshire, UK) that logs and processes the samples. An email reminder message to have a blood sample taken is sent 10 days after the sample kit has been posted. The helpdesk attempts to contact participants with outstanding blood samples on days 17 and 28 after the sample kit has been posted, and if the sample is not received after 31 days after the sample kit has been posted, blood sample results will be marked as missing data.

\section{Measures}

Table 2 provides details of the measures collected in the INFORM study and the stage of the study at which each is assessed. The primary outcome is objectively measured physical activity, defined as average acceleration $\left(\mathrm{m} / \mathrm{s}^{2}\right)$ over the observation period. Participants are instructed to continuously wear the accelerometer for 7 consecutive days and nights, and to carry on with all normal activities during this time. The accelerometers are waterproof and can be worn while swimming and showering. Accelerometers have been chosen as a measure of physical activity because they cause low participant burden and are not subject to the reporting bias or recall problems associated with the self-report methods [52].

The key secondary outcome, dietary behaviour, is objectively measured by levels of six serum carotenoids (alpha-carotene, beta-carotene, lutein, lycopene, betacryptoxanthin, zeaxanthin). Carotenoids are biologically active pigments in plants but are not synthesised in animals and as such they represent a valid biomarker of vegetable and fruit intake [53].

Other objectively measured secondary outcomes include total, HDL and LDL cholesterol and fructosamine. We also collect self-reported secondary outcomes via an online questionnaire. They include cardiovascular risk factors (self-reported weight, smoking status, alcohol consumption, physical activity and dietary behaviour); current medication and healthcare usage; perceived risk (perception of comparative and absolute risk); cognitive evaluation of provision of CHD risk scores (understanding, accuracy, acceptability); and psychological outcomes (anxiety associated with testing, fatalism, depression, stress and mood).

As with any other intervention, it is important to understand the mechanisms that explain why the provision of CVD risk information leads to change or not, and whether there are people with certain characteristics who may benefit more or less from CVD risk 
Table 2 INFORM measures at each stage of the study

\begin{tabular}{|c|c|c|c|c|c|c|}
\hline & Measure or Instrument name & INTERVAL & $\begin{array}{l}\text { INFORM } \\
\text { baseline }\end{array}$ & $\begin{array}{l}\text { INFORM } \\
\text { follow- } \\
\text { up }\end{array}$ & $\begin{array}{l}\text { Mode of } \\
\text { assessment }\end{array}$ & Note \\
\hline $\begin{array}{l}\text { Primary } \\
\text { outcome }\end{array}$ & Physical Activity Level* & $\checkmark$ & $\checkmark$ & $\checkmark$ & accelerometers & $\begin{array}{l}\text { using the Axivity AX3 - 3Axis logging } \\
\text { Accelerometer }\end{array}$ \\
\hline \multirow{21}{*}{$\begin{array}{l}\text { Secondary } \\
\text { outcomes }\end{array}$} & Traditional risk factors & & & & & \\
\hline & Fruit and vegetables intake & $\checkmark$ & & $\checkmark$ & carotenoids & \\
\hline & Weight & & $\checkmark$ & $\checkmark$ & self-report & as used in Godino et al. [56] and Knowles et al. [48] \\
\hline & $\begin{array}{l}\text { Cholesterol Panel (total, HDL } \\
\text { and LDL cholesterol, } \\
\text { triglycerides) }\end{array}$ & $\checkmark$ & & $\checkmark$ & blood serum & \\
\hline & Fructosamine & $\checkmark$ & & $\checkmark$ & blood serum & \\
\hline & $\begin{array}{l}\text { Nutrition Behaviour (fruit, } \\
\text { vegetables, whole grains, fish, } \\
\text { red and processed meat) }\end{array}$ & & $\checkmark$ & $\checkmark$ & self-report & $\begin{array}{l}\text { one-item questions that reflect the present } \\
\text { prevention guidelines on CVD }[16,10]\end{array}$ \\
\hline & Physical Activity Level & & $\checkmark$ & $\checkmark$ & self-report & EPIC-Norfolk Physical Activity Questionnaire [60] \\
\hline & Smoking Status & & $\checkmark$ & $\checkmark$ & self-report & \\
\hline & Alcohol Consumption & & $\checkmark$ & $\checkmark$ & self-report & \\
\hline & $\begin{array}{l}\text { Current Medication and } \\
\text { Healthcare Usage }\end{array}$ & & $\checkmark$ & $\checkmark$ & self-report & $\begin{array}{l}\text { adapted version of the Health Services Research } \\
\text { Unit Aberdeen questionnaire [61] }\end{array}$ \\
\hline & Perceived risk & & & & & \\
\hline & $\begin{array}{l}\text { Comparative and Absolute } \\
\text { Cardiovascular Risk }\end{array}$ & & $\checkmark$ & $\checkmark$ & self-report & $\begin{array}{l}\text { adapted according to Diefenbach et al. }[62] \text { and } \\
\text { used in other risk communication studies }[48,56]\end{array}$ \\
\hline & $\begin{array}{l}\text { Cognitive evaluation of provision } \\
\text { of coronary heart disease risk } \\
\text { scores }\end{array}$ & & & & & \\
\hline & Understanding of Risk Scores & & & $\checkmark$ & self-report & as used in another trial of risk communication [56] \\
\hline & $\begin{array}{l}\text { Perceived Accuracy of Risk } \\
\text { Scores }\end{array}$ & & & $\checkmark$ & self-report & $\begin{array}{l}\text { as used in other risk communication studies } \\
{[48,56]}\end{array}$ \\
\hline & $\begin{array}{l}\text { Acceptability of the } \\
\text { Intervention }\end{array}$ & & & $\checkmark$ & self-report & in line with the Heart to Health study [50] \\
\hline & Psychological outcomes & & & & & \\
\hline & Stress & & $\checkmark$ & $\checkmark$ & self-report & $\begin{array}{l}\text { as used in the Randomized Trial of Personal } \\
\text { Genomics for preventive cardiology [48] }\end{array}$ \\
\hline & Mood & & $\checkmark$ & $\checkmark$ & self-report & adapted from the Patient Health Questionnaire [63] \\
\hline & $\begin{array}{l}\text { Coronary Heart Disease-related } \\
\text { Worry }\end{array}$ & & $\checkmark$ & $\checkmark$ & self-report & $\begin{array}{l}\text { an adaptation of the Cancer Related Worry } \\
\text { Scale [64] }\end{array}$ \\
\hline & $\begin{array}{l}\text { Genetic Risk-related Worry/ } \\
\text { Anxiety }\end{array}$ & & $\checkmark$ & & self-report & as used by Knowles et al. [48] \\
\hline \multirow{7}{*}{$\begin{array}{l}\text { Moderators } \\
\text { and } \\
\text { mediators }\end{array}$} & $\begin{array}{l}\text { Sociodemographic } \\
\text { characteristics }\end{array}$ & & & & & \\
\hline & Sex, Age, Ethnicity & $\checkmark$ & & & self-report & \\
\hline & Marital Status & & $\checkmark$ & & self-report & \\
\hline & $\begin{array}{l}\text { Socioeconomic Status (level of } \\
\text { education, level of income, } \\
\text { living area) }\end{array}$ & & $\checkmark$ & & self-report & $\begin{array}{l}\text { adapted from the European Health Interview } \\
\text { Survey; deprivation level of the area based on the } \\
\text { Index of Multiple Deprivation, Department for } \\
\text { Communities and Local Government }\end{array}$ \\
\hline & Numeracy Skills & & $\checkmark$ & & self-report & 3-item Schwartz scale [65] \\
\hline & $\begin{array}{l}\text { Family History of Coronary } \\
\text { Heart Disease }\end{array}$ & & $\checkmark$ & & self-report & \\
\hline & History of Genetic Testing & & $\checkmark$ & & self-report & \\
\hline
\end{tabular}


Table 2 INFORM measures at each stage of the study (Continued)

\begin{tabular}{|c|c|c|c|c|}
\hline $\begin{array}{l}\text { History of Coronary Heart } \\
\text { Disease Risk Assessment }\end{array}$ & $\checkmark$ & & self-report & \\
\hline Self-rated Health & $\checkmark$ & $\checkmark$ & self-report & Ware et al. [66] \\
\hline $\begin{array}{l}\text { Barriers to risk-reducing } \\
\text { strategy }\end{array}$ & $\checkmark$ & & self-report & in line with the Heart to Health study [50] \\
\hline \multicolumn{5}{|l|}{$\begin{array}{l}\text { Cognitive and emotional } \\
\text { theory-based antecedents to } \\
\text { behaviour change }\end{array}$} \\
\hline Intentions & $\checkmark$ & & self-report & $\begin{array}{l}\text { adapted according to Ajzen [67] and used in } \\
\text { previous similar research [56] }\end{array}$ \\
\hline Perception of Diet & $\checkmark$ & & self-report & \\
\hline Perception of Physical Activity & $\checkmark$ & & self-report & \\
\hline $\begin{array}{l}\text { Coronary heart disease risk } \\
\text { Representations }\end{array}$ & $\checkmark$ & & self-report & the Brief Illness Perceptions Questionnaire [68] \\
\hline Self-efficacy & $\checkmark$ & & self-report & as used in previous behavioural research $[56,68]$ \\
\hline Response Efficacy & $\checkmark$ & $\checkmark$ & self-report & as used in previous behavioural research $[52,69]$ \\
\hline Social Support & $\checkmark$ & & self-report & $\begin{array}{l}\text { the Multidimensional Scale of Perceived Social } \\
\text { Support [70] }\end{array}$ \\
\hline Time Orientation & $\checkmark$ & & self-report & as used in a study by Peretti-Watel et al. [71] \\
\hline Sense of Coherence & $\checkmark$ & & self-report & 3-items scale, Lundberg et al. [72] \\
\hline Comparative Optimism & $\checkmark$ & & self-report & comparative perceived risk \\
\hline
\end{tabular}

*participants who did not take part in physical activity monitoring during the INTERVAL Study will be required to undertake baseline activity monitoring over 7 days

communication. To understand this, the following potential moderators and mediators of the relationship between provision of risk information and behaviour change are being measured as part of the INFORM study: a) sociodemographic characteristics (ethnicity, socioeconomic status, education, income, numeracy skills and marital status); b) history of genetic testing and CHD risk assessment; c) self-rated health; d) cognitive and emotional theory-based antecedents to behaviour change (behavioural intentions, perception of diet and physical activity, beliefs about CVD, self-efficacy and response efficacy, social support, mood and personality characteristics); and f) personal barriers to risk reducing strategies.

\section{Qualitative data collection Qualitative interviews}

An experienced qualitative researcher conducts in-depth individual interviews with a sub-sample of trial participants starting soon after the randomisation and finishing around 2-3 months after the 3-month follow-up period is complete. Interviews cover issues related to the interviewees' experience of taking part in the trial; more specifically, their understanding of CHD risk, factors affecting their understanding, reaction to receiving information about risk and lifestyle advice, the extent to which understanding of risk contributes to motivation for behaviour change, the implementation of intention to change after receiving risk information and lifestyle advice, and the facilitators and barriers for such a change. The qualitative study focuses mainly on participants in the intervention arms because the lack of an intervention leaves little to discuss and explore qualitatively with the members of the control arm. However, in order to explore the impact of receiving different types of information in different stages, we aim to have up to six interviews with members of arms who initially received no intervention/only lifestyle advice/only phenotypic risk score, after they receive all their risk scores at the end of the intervention.

In order to supplement the quantitative data which is collected at only two time points (at baseline and 12 weeks later), we aim to interview different participants at different time points, interviewing some immediately after the randomisation, others half way through the intervention and others towards the end of the intervention or up to 3 months after it ends. Proportionally more participants whose risk score is medium or high are purposively recruited as this is the population most likely to have a stronger motivation to change their lifestyle. A subset of this group may be interviewed more than once - for example immediately after the start of intervention and then after the completion of the intervention in order to explore early expectations/motivation and compare them with later perceptions or behaviour. For the participants who do not express an intention to 
change their lifestyle and those who are approached at later stages of the intervention, the discussion on the reaction to receiving the risk scores or lifestyle advice is conducted retrospectively focusing on the period from receiving the risk scores and up to the point of the interview. Interviewees are asked to sign an interview consent form giving permission for their interview to be recorded.

\section{Qualitative focus groups}

Two to three focus groups are conducted at different points of time throughout the trial. Approximately 8-12 participants take part in each of the focus groups. These participants do not take part in the individual qualitative interviews. The focus groups are facilitated by an experienced qualitative researcher and one or two assistants from the research team. Participants are purposively selected in order to include people with various demographic backgrounds. However, at least two of the groups may be selected for gender homogeneity (male/ female-only) in order to encourage greater participation in what may be considered a gender-sensitive issue. The focus groups cover issues related to the relationship between risk awareness, motivation for change and actual change of behaviour and lifestyle, but their main focus is on communication and understanding of CHD risk estimates as well as the lifestyle advice. We hope to benefit from the interactive aspect of the focus groups in order to gain as rich as possible feedback regarding the advantages and disadvantages of the forms of communication of risk and lifestyle advice used in the trial. Permission is sought from the participants, as outlined in the consent process, for the focus groups to be recorded and transcribed.

\section{Withdrawal}

A participant is free to withdraw their consent from the study, at any time, without giving a reason. All requests from participants to withdraw from the study are directed to the study coordination team who will discuss the options for withdrawal. The study data manager places a flag on the research database to ensure appropriate use of data and notifies the study coordination team of withdrawal. University of Cambridge research staff instructs the central laboratory to remove all archive samples from the biorepository for participants who have been withdrawn from the study and who have requested no further use of their stored samples.

\section{Adverse events}

This is a low-risk trial with little reason to consider that adverse events would arise as a result of following any of the interventions. Accordingly, no formal adverse event monitoring is planned. However, events may incidentally come to the attention of the research team (e.g. via interviews or focus groups, or direct communication with participants via the study coordination team) which will be recorded and followed up accordingly.

\section{Statistical analyses}

Sample size calculation - randomised controlled trial

The primary outcome is between-group differences in the change (follow-up minus baseline) of the average acceleration $\left(\mathrm{m} / \mathrm{s}^{2}\right)$ over the observation period, measured using the Axivity AX3 3-Axis Logging Accelerometer ${ }^{\circ}$. The intervention will be potentially important if it increases the primary outcome by $10 \%$. This corresponds to an additional $10 \mathrm{~min}$ of brisk walking per day or an additional $3 \mathrm{~min}$ of jogging per day. The same effect size was used in the Understanding Risk trial by Price et al. [54] among people at high risk of CVD with one month of follow-up. This effect size is also in line with the ProActive trial that aimed to increase physical activity among individuals at high risk of type 2 diabetes [55]. To allow for four pairwise comparisons in the primary analysis, the significance level was set to $1.25 \%$. We calculated that 186 participants per group will be needed to detect this effect with $80 \%$ power and $95 \%$ confidence, assuming the estimated standard deviation (SD) of change in physical activity from baseline to follow-up is 0.05 and the correlation between physical activity at baseline and follow-up is 0.6 [56]. Allowing for an attrition rate of $20 \%$, we therefore aim to randomise a total of 932 participants (233 per group).

\section{Sample size - qualitative study}

A sample size of between 30 and 35 male and female participants will be selected purposively, mainly from the intervention arms to include people with various demographic backgrounds. This sample size is common for this kind of study $[57,58]$ and is consistent with the time resources required for this type of data analysis and the predicted range of number of interviews required for achieving saturation.

\section{Analysis design - randomised controlled trial}

All trial analyses will be based on the Intention To Treat (ITT) principle. Those with missing follow-up data will be excluded from the analyses (a complete case approach). We will perform sensitivity analyses to assess the impact of missing follow-up data on the results. Participants with missing baseline data will be included in the analyses using the missing-indicator method [59].

The null hypothesis for the primary analysis is that there is no difference between any of Groups 1 to 4 . Estimates of the following pairwise differences will be calculated: 1) Group 1 (control group) vs. Group 2 (lifestyle advice only) - to estimate the effect of providing lifestyle advice compared with no intervention; 2) Group 3 
(phenotypic risk score and lifestyle advice) vs. Group 4 (phenotypic and genetic risk scores and lifestyle advice) - to estimate the effect of providing a genetic risk score in addition to lifestyle advice and a phenotypic risk score; 3) Group 3 + Group 4 vs. Group 1 - to estimate the effect of providing risk score information and lifestyle advice compared with no intervention, and 4) Group 3 + Group 4 vs. Group 2 - to estimate the effect of providing risk score information in addition to lifestyle advice.

Mean changes in objectively measured physical activity between baseline and 3-month follow-up will be analysed using analysis of covariance (ANCOVA). The ANCOVA model will model change (follow-up minus baseline) in objectively measured physical activity, adjusted for baseline. A test involving three degrees of freedom (3 d.f.) will be performed of the null hypothesis that there is no difference between the four randomised groups. If the $\mathrm{p}$-value from this test is $<0.01$, this will imply that there are differences between the groups. The ANCOVA model will also be used to derive estimates of the differences in mean change and $95 \%$ confidence intervals for each of the four pairwise comparisons described above. To avoid detracting the focus of the results from the overall 3 d.f. test, $p$-values for each of the pairwise comparisons will not be calculated.

For each continuous secondary endpoint, the four pairwise differences will be estimated, together with $95 \%$ confidence intervals, using ANCOVA as described for the primary outcome analyses (except Coronary Heart Disease-related Worry). The Coronary Heart Disease-related Worry will be measured only at followup, so the four pairwise differences will be estimated, together with $95 \%$ confidence intervals, using linear regression. A 3 d.f. test will be performed of the null hypothesis that there is no difference between the four randomised groups. For categorical endpoints, similar analyses will be performed using logistic and multinomial regression models.

\section{Subgroup analyses}

For the primary endpoint, the ANCOVA model will be extended to include an interaction between the intervention effect and (1) age (continuous variable), (2) baseline phenotypic CHD risk score (continuous variable) and (3) sex. If the $\mathrm{p}$-value for interaction is $<0.05$, then the number and percentage of individuals with the endpoint within each randomised group, together with intervention effect (and $95 \%$ confidence intervals) will be reported separately within categories defined by the subgroup variable. For age and baseline CHD risk score, the cut-off for the categories will be their median values at baseline.

\section{Multiplicity}

Given the number of endpoints, randomised groups and therefore comparisons, the results for the primary endpoint will be regarded as convincing if the $p$-value from the 3 d.f. test is $<0.01$, while the results for each secondary endpoint will be regarded as convincing if the relevant $p$-value from the 3 d.f. test is $<0.001$.

\section{Qualitative analyses}

Thematic analysis will be used to analyse the qualitative data. The transcriptions will be coded using NVivo software. To increase the rigour and validity of the analysis, and as a form of triangulation, the framework development (coding tree) will be conducted by a subgroup of three members of the team (the lead qualitative researcher and two others). In addition, a subset of $20 \%$ of transcripts will be double-coded by two members of the research team, and disagreements will be discussed in order to reach consensus about interpretation and indexing. Analysis will involve two stages:

(1)Data management which will include: a) familiarisation with the data, including reading transcripts and notes and/or listening to the audio dialogue in order to extract main themes and ideas; b) thematic framework development, identifying the key issues and concepts present in the data and creating a coding tree which will be conducted both inductively, based on the data, and deductively, based on the research questions; c) indexing the data.

(2)Interpretation stage which will include focused or axial coding, defining the main concepts and mapping the ways in which different parts of the data are related to each other.

\section{Discussion}

The INFORM randomised trial has been designed to contribute toward the understanding of the impact of provision of phenotypic and genetic CHD risk information on health-related behaviour change and other important clinical outcomes. Comparison between randomised trial arms will enable estimation of: 1 ) the effect of providing web-based lifestyle advice; 2 ) the effect of providing a genetic risk score in addition to lifestyle advice and a phenotypic risk score; 3) the effect of providing risk score information and lifestyle advice and 4) the effect of providing risk score information in addition to lifestyle advice. The INFORM study will also provide information on potential moderators and mediators between the intervention and health-related behaviour change and other clinical outcomes.

Even though the routine use of CVD risk scores is strongly advocated by most cardiovascular guidelines 
$[10,11]$, relatively little is reliably known about the benefits of the provision of different forms of CVD risk scores to individuals and about any potential harms (such as the possibility of increased anxiety or false reassurance). Previous systematic reviews [18, 19] have concluded that no robust recommendations can be made about the value of providing CVD risk information owing to limitations in previous studies, including lack of adequate power and lack of objective measures of health-related behaviours.

The INFORM study is well-placed to advance current knowledge on this topic because it is a trial that combines several advantages, including: a) power to identify clinically important differences between groups in this study's principal outcomes; b) objective measures of health-related behaviours; c) simplicity of the interventions and relevance to primary care. If the intervention(s) in this trial prove to be effective, then the components of the intervention should be relatively easy to implement in primary care practice. Hence, the INFORM trial should provide a reliable evaluation of the impact of the provision of CVD risk information, thereby potentially informing policy decisions and guiding clinical practice about the routine use of CVD risk scores in primary care.

\section{Research governance Ethical approval}

Ethical approval was received from NRES Committee East of England - Cambridge Central (14/EE/1164) on 03/12/2014. NHS Research and Development assurance has been received by our lead CCG (Cambridgeshire and Peterborough CCG) and CRN: Eastern. The trial was prospectively registered at the ISRCTN Registry (ISRCTN17721237) on 12/01/ 2015. This paper details version 1.2 of the trial protocol dated 13/01/2015. The trial has been adopted by the NIHR Portfolio.

\section{Study sponsor}

The University of Cambridge is the sponsor of this trial.

\section{Data handling and quality assurance}

A Data Monitoring Committee is not considered appropriate for this trial considering that the likely risks to participants are known to be minimal, recruitment and follow-up are over a short period of time, and the data for this trial is both collected and stored online and, as such, is expected to have a minimal error rate. Any incidental safety information will be reported to the Trial Steering Committee (TSC). The Data Manager is responsible for the quality and integrity of data and will provide a data report to each TSC meeting. Data are stored in accordance with the University of Cambridge School of Clinical Medicine Information Governance policy which relates to sensitive/ identifiable personal information collected and stored for the purposes of research.

\section{Patient and public involvement (PPI)}

Two representatives from the PPI Panel at Cambridge University Hospitals NHS Foundation Trust were recruited in June 2014. PPI representatives have played an integral role in the INFORM study and contributed to the different stages of the research. More specifically, PPI representatives commented on the protocol, questionnaires, wording and format of the presentation of the risk score estimates, critically revised patient information sheets (both qualitative and quantitative) as well as the web-based lifestyle intervention. PPI representatives have a central role in the TSC. We expect that they will also be involved with developing newsletters and taking part in dissemination activities.

\section{Trial oversight}

In addition to the Research Ethics Committee, the INFORM study is overseen by the TSC and Trial Management Group (TMG). The purpose of the TSC is to provide oversight of the clinical trial, with particular reference to adherence to the protocol, compliance with the Department of Health Research Governance Framework and the Guidelines for Good Clinical Practice, and the rights, safety and wellbeing of the trial participants. In addition to members of the research team, the Committee contains a number of independent members, specifically the Independent Chair, Independent Academics and PPI representatives. The purpose of the TMG is to monitor the objectives and progress of the trial, oversee day-to-day management of the study, consider any new information that may be relevant to the trial and discuss any scientific matters contained in, or relevant to, the trial. It includes investigators, the trial manager and project administrative assistant.

\section{Research dissemination and data preservation for sharing}

The investigators will analyse data according to a predefined analysis plan in a timely manner. Members of the research team will be involved in reviewing drafts of the manuscripts, abstracts, and any other publications arising from the trial. The Chief Investigator will have final approval on all publications and press releases. Authorship of publications will be determined by ICMJE guidelines. A lay summary of the research findings will be available on the study website [29] and study participants will be notified by email when they are available.

The database manager will take responsibility for data curation and archiving, and all data sets will be kept securely with no access from unauthorised personnel. Data will be stored so that it can be accessed, used and understood by subsequent users. On completion of the data 
collection we will develop a data dictionary so that scientists can request release of a study dataset from the investigators. When the investigators have completed their planned analyses, the anonymised data will be made available for use by others and will be shared under appropriate data sharing agreements. Primary data and the Trial Master File will be retained securely in their original form for a minimum of 10 years.

\section{Trial status}

Ongoing. Recruitment was completed in June 2015.

\section{Additional file}

\section{Additional file 1: Appendix A. An example of presentation of}

phenotypic coronary heart disease risk score. Appendix B. Mathematical coronary heart disease functions to predict 10-year risk of coronary heart disease. Appendix C. An example of presentation of genetic coronary heart disease risk score. Appendix D. INFORM SNPs for genetic risk score. Appendix E. Mathematical formulas for calculation of risk estimates based on genetic risk score (GRS). (ZIP $112 \mathrm{~kb}$ )

\section{Abbreviations}

ANCOVA: analysis of covariance; BMI: body mass index; CHD: coronary heart disease; CVD: cardiovascular disease; GP: general practitioner; GRS: genetic risk score; InGRS: natural log-transformed genetic risk score; HDL: high-density lipoprotein; ITT: Intention to Treat principle; LDL: Iow-density lipoprotein; NHS: the National Health Service; NHSBT: NHS Blood and Transplant; NICE: National Institute for Health and Care Excellence; PPI: Patient and public involvement; SD: standard deviation; SNP: single nucleotide polymorphism; TMG: Trial Management Group; TSC: Trial Steering Committee; 3 d.f: 3 degrees of freedom test.

\section{Competing interests}

The authors declare that they have no competing interests.

\section{Authors' contributions}

SG is the Chief Investigator of the INFORM trial. JD is the co-chief investigator of the INTERVAL trial (which provides the framework from which the participants in the INFORM trial are recruited) and he is the principal investigator of EPIC-CVD, a pan-European consortium which involves a series of studies, including INFORM, to help develop and evaluate approaches to CVD risk assessment and screening. BS and SG developed the research questions, measures and designed the trial. BS, GS, RAP and SG developed the interventions. SJS is the chief statistician and will conduct the primary statistical analyses. $J \mathrm{~L}$ is the trial manager. MW is the senior data manager. GS leads the qualitative component of the study. BS, JL, ASB, EDA CG, KL, SM, CM, RAP, GS, ZT, JUS, JD, SG have contributed to the conception, design and execution of the trial and have read and approved this submitted version of the manuscript.

\section{Acknowledgements}

NHS Blood and Transplant, the sponsor of the INTERVAL trial, assisted with the involvement of blood donors in INFORM. A complete list of the investigators and contributors to the INTERVAL trial is provided in reference [24]. The web-based lifestyle intervention for CHD prevention is based on one that was originally developed for the Heart to Health study (http://hpdp. unc.edu/research/projects/heart-to-health/). In addition some materials were originally developed by Leicester Diabetes Centre (http://www.leicesterdiabetescentre.org.uk). Affymetrix (Santa Clara, US) provided genotyping. The MRC Epidemiology Unit Physical Activity Team provided expertise. UK Biocentre (Stockport, UK) provided laboratory support. Vitas (Oslo, Norway) conducted assays. We would like to acknowledge contribution of Rebecca Simmons to the protocol development and would like to thank to Simon Thompson and Michael Sweeting for their helpful comments concerning statistical issues. We would like to express our gratitude to Vivienne Shaw, Emily Ikelle and Alexander Phillips from NHS Research and Development for their help. Lastly we would like to thank to all participants who agreed to take part in INFORM trial.

\section{Funding}

INFORM is funded by European Commission Framework 7 EPIC-CVD Grant agreement no: 279233. NHS Blood and Transplant funded the INTERVAL trial. DNA extraction and genotyping in INTERVAL/INFORM was funded by the UK National Institute of Health Research. The coordinating team for INTERVAL/INFORM at the Cardiovascular Epidemiology Unit of the University of Cambridge was supported by core funding from: UK Medical Research Council (G0800270), British Heart Foundation (SP/09/002), British Heart Foundation Cambridge Cardiovascular Centre of Excellence, and UK National Institute for Health Research Cambridge Biomedical Research Centre.

\section{Trial committees and teams}

Trial Steering Committee: Adam Butterworth (Senior Investigator), Emanuele Di Angelantonio (Senior Investigator), Chris Girling (Independent Member, Patient and Public Involvement representative), Simon Griffin (Chief Investigator), Sheila Hardy (Independent Academic Member,

Northamptonshire Healthcare NHS Foundation Trust), Richard Houghton (Head of Cardiovascular Epidemiology Unit Operations), Kathryn Lawrence (Independent Member, Patient and Public Involvement representative), Joanne Lucas (Scientific Research Coordinator), Tom Marshall (Chair and Independent Academic Member, University of Birmingham), Carmel Moore (Senior Investigator and Senior Scientific Coordinator of the INTERVAL trial), Rupert Payne (Investigator), Carolyn Read (Sponsor Representative), Stephen Sharp (Chief Statistician), Guy Shefer (Investigator), Barbora Silarova (Investigator), Zoe Tolkien (Investigator and Scientific Research Coordinator of the INTERVAL trial), Juliet Usher-Smith (Investigator), Matthew Walker (Senior Data Manager).

Trial Management Committee: Adam Butterworth, Emanuele Di

Angelantonio, Simon Griffin (Chair), Joanne Lucas, Stuart Mackintosh, Carme Moore, Rupert Payne, Guy Shefer, Barbora Silarova, Zoe Tolkien, Juliet Usher-Smith, Matthew Walker (previous member: Rebecca Simmons). Data Management Team: Sarah A Spackman, Matthew Walker. INFORM Helpdesk: Susan Burton, Victoria Clarke, Rachel Henry, Stuart Mackintosh.

EPIC-CVD scientific coordinating centre: Adam Butterworth, John Danesh, Richard Houghton, Sarah A Spackman.

UK Biocentre: Kathrine Bancroft, Adam King, Cedric Mochon, Nicholas Oates, Christine Ryan, Kristian Spreckley, Joanna Williams.

\section{Author details}

MRC Epidemiology Unit, University of Cambridge, Institute of Metabolic Science, Cambridge CB2 OQQ, UK. ${ }^{2}$ Cardiovascular Epidemiology Unit, Department of Public Health and Primary Care, Strangeways Research Laboratory, Wort's Causeway, Cambridge CB1 8RN, UK. ${ }^{3}$ The INTERVAL trial coordinating centre, Department of Public Health and Primary Care, Strangeways Research Laboratory, Wort's Causeway, Cambridge CB1 8RN, UK. ${ }^{4}$ Patient and Public Involvement representatives, Cambridge, UK. ${ }^{5}$ Cambridge Centre for Health Services Research, University of Cambridge, Institute of Public Health, Forvie Site, Robinson Way, Cambridge CB2 OSR, UK. ${ }^{6}$ The Primary Care Unit, Department of Public Health and Primary Care, University of Cambridge, Strangeways Research Laboratory, 2 Wort's Causeway, Cambridge CB1 8RN, UK.

Received: 29 July 2015 Accepted: 26 August 2015 Published online: 07 September 2015

\section{References}

1. World Health Organization. Global status report on noncommunicable diseases 2010. 2011. http://whqlibdoc.who.int/publications/2011/ 9789240686458_eng.pdf?ua=1. Accessed 5 May 2014.

2. Mendis S, Puska P, Norrving B. Global atlas on cardiovascular disease prevention and control. World Health Organization. 2011. http:// whqlibdoc.who.int/publications/2011/9789241564373_eng.pdf. Accessed 5 May 2014 
3. World Health Organization. The top 10 causes of death 2013 Fact sheet no. 310. 2013. http://www.who.int/mediacentre/factsheets/fs310/en/. Accessed 25 April 2014

4. Mathers CD, Loncar D. Projections of global mortality and burden of disease from 2002 to 2030. PLoS Med. 2006;3(11), e442.

5. Anderson KM, Odell PM, Wilson PW, Kannel WB. Cardiovascular disease risk profiles. Am Heart J. 1991;121(1 Pt 2):293-8.

6. Hippisley-Cox J, Coupland C, Vinogradova Y, Robson J, May M, Brindle P. Derivation and validation of QRISK, a new cardiovascular disease risk score for the United Kingdom: prospective open cohort study. BMJ. 2007:335(7611):136

7. Woodward M, Brindel P, Tunstall-Pedoe H. Adding social deprivation and family history to cardiovascular risk assessment: the ASSIGN score from the Scottish Heart Health Extended Cohort (SHHEC). Heart. 2007;93(2):172-6.

8. Conroy RM, Pyorala K, Fitzgerald AP, Sans S, Menotti A, De BG, et al. Estimation of ten-year risk of fatal cardiovascular disease in Europe: the SCORE project. Eur Heart J. 2003;24(11):987-1003.

9. Ridker PM, Buring JE, Rifai N, Cook NR. Development and validation of improved algorithms for the assessment of global cardiovascular risk in women: the Reynolds Risk Score. JAMA. 2007;297(6):611-9.

10. JBS3 Board. Joint British Societies 'consensus recommendations for the prevention of cardiovascular disease (JBS3). Heart. 2014;100 Suppl 2:ii1-ii67.

11. Goff Jr DC, Lloyd-Jones DM, Bennett G, O'Donnell CJ, Coady S, Robinson J, et al. ACC/AHA guideline on the assessment of cardiovascular risk: a report of the American College of Cardiology/American Heart Association task force on practice guidelines. Circulation. 2014;129(25 Suppl 2):S49-73.

12. NHS Employers and British Medical Association Quality and Outcomes Framework Guidance for GMS Contract 2011-12. London: The NHS Confederation (Employers) Company; 2011.

13. Krogsbø\|ll LT, Jørgensen KJ, Larsen CG, Gøtzsche PC. General health checks in adults for reducing morbidity and mortality from disease: Cochrane systematic review and meta-analysis. BMJ. 2012;345, e7191.

14. Satish M. Coronary heart disease in clinical practice. London: Goldaming, Springer; 2005

15. Gaziano M, Ridker PM, Libby P. Primary and secondary prevention of coronary heart disease. In: Bonow RO, Mann DL, Zipes DP, Libby P, editors. Braunwald's heart disease. A textbook of cardiovascular medicine. Philadelphia: Elsevier Saunders; 2012. p. 1010-35.

16. Rabar S, Harker M, O'Flynn N, Wierzbicki AS, Guideline Development Group. Lipid modification and cardiovascular risk assessment for the primary and secondary prevention of cardiovascular disease: summary of updated NICE guidance. BMJ. 2014;349:94356.

17. National Institute for Health and Care Excellence. Prevention of cardiovascular disease $(\mathrm{PH} 25)$ - review proposal. Centre for Public Health Excellence. 2010. http://www.nice.org.uk/guidance/ph25/ documents/prevention-of-cardiovascular-disease-review-decision2. Accessed 25 April 2014

18. Brindle P, Beswick A, Fahey T, Ebrahim S. Accuracy and impact of risk assessment in the primary prevention of cardiovascular disease: a systematic review. Heart. 2006;92(12):1752-9.

19. Sheridan SL, Viera AJ, Krantz MJ, Ice CL, Steinman LE, Peters KE, et al. The effect of giving global coronary risk information to adults: a systematic review. Arch Intern Med. 2010;170(3):230-9.

20. Wang C, Bowen DJ, Kardia SL. Research and practice opportunities at the intersection of health education, health behaviour and genomics. Health Educ Behav. 2005;32(5):686-701.

21. Institute of Medicine. Initial National priorities for Comparative Effectiveness research. Washington, DC: Institute of Medicine; 2009.

22. Rosenstock IM. Adoption and maintenance of lifestyle modifications. Am J Prev Med. 1988:4(6):349-52.

23. Marteau TM, French DP, Griffin SJ, Prevost AT, Sutton S, Watkinson C, et al. Effects of communicating DNA-based disease risk estimates on risk-reducing behaviours. Cochrane Database Syst Rev. 2010;2010(10):CD007275

24. Moore C, Sambrook J, Walker M, Tolkien Z, Kaptoge S, Allen D, et al. The INTERVAL trial to determine whether intervals between blood donations can be safely and acceptably decreased to optimise blood supply: study protocol for a randomised controlled trial. Trials. 2014;15:363.

25. NHS Blood and Transplant criteria for giving blood, http://www.blood.co.uk/ giving-blood/who-can-give-blood/. Accessed 25 April 2014.
26. INFORM Study. http://www.informstudy.org.uk/index.htm. Accessed 20 April 2015.

27. Persell SD, Lloyd-Jones DM, Friesema EM, Cooper AJ, Baker DW. Electronic health record-based patient identification and individualized mailed outreach for primary cardiovascular disease prevention: a cluster randomized trial. J Gen Intern Med. 2013;28(4):554-60.

28. Payne RA. Cardiovascular risk. Br J Clin Pharmacol. 2012;74(3):396-410.

29. D'Agostino Sr RB, Vasan RS, Pencina MJ, Wolf PA, Cobain M, Massaro JM, et al. General cardiovascular risk profile for use in primary care: the Framingham Heart Study. Circulation. 2008;117(6):743-53.

30. Soureti A, Hurling R, Murray P, van Mechelen W, Cobain M. Evaluation of a cardiovascular disease risk assessment tool for the promotion of healthier lifestyles. Eur J Cardiovasc Prev Rehabil. 2010;17(5):519-23.

31. Carlsson AC, Wandell PE, Gigante B, Leander $K$, Hellenius ML, de Faire U. Seven modifiable lifestyle factors predict reduced risk for ischemic cardiovascular disease and all-cause mortality regardless of body mass index: A cohort study. Int J Cardiol. 2013;168(2):946-52.

32. Akesson A, Weismayer C, Newby PK, Wolk A. A combined effect of low-risk dietary and lifestyle behaviours in primary prevention of myocardial infarction in women. Arch Intern Med. 2007;167(19):2122-7.

33. Khaw KT, Wareham N, Bingham S, Welch A, Luben R, Day N. Combined impact of health behaviours and mortality in men and women: the EPIC-Norfolk prospective population study. PLoS Med. 2008:5(1), e12.

34. NHS choices. 5 A DAY. http://www.nhs.uk/LiveWell/5ADAY/Pages/ 5ADAYhome.aspx. Accessed 25 April 2014

35. SACN Advice on Fish Consumption. 2004. https://www.gov.uk/government/ publications/sacn-advice-on-fish-consumption. Accessed 25 April 2014.

36. World Cancer research Fund. Red and processed meat and cancer prevention. http://www.wcrf-uk.org/uk/preventing-cancer/ ways-reduce-cancer-risk/red-and-processed-meat-and-cancer-prevention. Accessed 25 April 2014.

37. Day N, Oakes S, Luben R, Khaw KT, Bingham S, Welch A, et al. EPIC-Norfolk: study design and characteristics of the cohort. European Prospective Investigation of Cancer. Br J Cancer. 1999;80 Suppl 1:95-103.

38. CARDIoGRAMplusC4D Consortium, Deloukas P, Kanoni S, Willenborg C, Farrall M, Assimes TL. Large-scale association analysis identifies new risk loci for coronary artery disease. Nat Genet. 2013;45(1):25-33.

39. EPIC-CVD. Individualised CVD risk assessment across Europe. http://www.epiccvd.eu/. Accessed 25 April 2014.

40. Schunkert $H$, König IR, Kathiresan S, Reilly MP, Assimes TL, Holm H, et al. Large-scale association analysis identifies 13 new susceptibility loci for coronary artery disease. Nat Genet. 2011;43(4):333-8.

41. Coronary Artery Disease (C4D) Genetics Consortium. A genome-wide association study in Europeans and South Asians identifies five new loci for coronary artery disease. Nat Genet. 2011:43(4):339-44

42. IBC 50K CAD Consortium. Large-scale gene-centric analysis identifies novel variants for coronary artery disease. PLoS Genet. 2011;7(9):e1002260.

43. Fair AK, Murray PG, Thomas A, Cobain MR. Using hypothetical data to assess the effect of numerical format and context on perception of coronary heart disease risk. Am J Health Promot. 2008;22(4):291-6.

44. Lipkus IM, Samsa G, Rimer BK. General performance on a numeracy scale among highly educated samples. Med Decis Making. 2001;21(1):37-44.

45. Kirby M, Machen L. Impact on clinical practice of the Joint British Societies' cardiovascular risk assessment tools. Int J Clin Pract. 2009;63(12):1683-92.

46. Lopez-Gonzalez AA, Aguilo A, Frontera M, Bennasar-Veny M, Campos I, Vicente-Herrero T, et al. Effectiveness of the Heart Age tool for improving modifiable cardiovascular risk factors in a Southern European population: a randomized trial. Eur J Prev Cardiol. 2015;22(3):389-96.

47. Hill S, Spink J, Cadilhac D, Edwards A, Kaufman C, Rogers S, et al. Absolute risk representation in cardiovascular disease prevention: comprehension and preferences of health care consumers and general practitioners involved in a focus group study. BMC Public Health. 2010;10:108

48. Knowles JW, Assimes TL, Kiernan M, Pavlovic A, Goldstein BA, Yank V, et al Randomized trial of personal genomics for preventive cardiology: design and challenges. Circ Cardiovasc Genet. 2012;5(3):368-76.

49. Sheridan SL, Behrend L, Vu MB, Meier A, Griffith JM, Pignone MP. Individuals' responses to global CHD risk: a focus group study. Patient Educ Couns. 2009;76(2):233-9.

50. Sheridan SL, Draeger LB, Pignone MP, Sloane PD, Samuel-Hodge C, Finkelstein EA, et al. Designing and implementing a comparative 
effectiveness study of two strategies for delivering high quality CHD prevention: methods and participant characteristics for Heart to health study. Contemp Clin Trials. 2013;36(2):394-405.

51. Sheridan SL, Draeger LB, Pignone MP, Keyserling TC, Simpson Jr RJ, Rimer B, et al. A randomized trial of an intervention to improve use and adherence to effective coronary heart disease prevention strategies. BMC Health Serv Res. 2011;11:331.

52. Trost SG, O' Neil M. Clinical use of objective measures of physical activity. Br J Sports Med. 2014;48(3):178-81.

53. Rock CL, Flatt SW, Natarajan L, Thomson CA, Bardwell WA, Newman VA, et al. Plasma carotenoids and recurrence-free survival in women with a history of breast cancer. J Clin Oncol. 2005;23(27):6631-8.

54. Price $\mathrm{CH}$, Tucker L, Griffin SJ, Holman RR. The impact of individualised cardiovascular disease (CVD) risk estimates and lifestyle advice on physical activity in individuals at high risk of CVD: a pilot $2 \times 2$ factorial understanding risk trial. Cardiovasc Diabetol. 2008;7:21.

55. Ekelund U, Griffin SJ, Wareham NJ. Physical activity and metabolic risk in individuals with a family history of type 2 diabetes. Diabetes Care. 2007:30(2):337-42.

56. Godino JG, van Sluijs EMF, Marteau TM, Sutton S, Sharp SJ, Griffin SJ. Effect of communicating genetic and phenotypic risk for type 2 diabetes in combination with lifestyle advice on objectively measured physical activity protocol of a randomised controlled trial. BMC Public Health. 2012;12:444.

57. Middlemass JB, Yazdani MF, Kai J, Standen PJ, Qureshi N. Introducing genetic testing for cardiovascular disease in primary care: a qualitative study. Br J Gen Pract. 2014;64(622):e282-9.

58. Meyer SB, Coveney J, Ward PR. A qualitative study of CVD management and dietary changes: problems of 'too much' and 'contradictory' information. BMC Fam Pract. 2014;15:25

59. White IR, Thompson SG. Adjusting for partially missing baseline measurements in randomized trials. Stat Med. 2005;24(7):993-1007.

60. Wareham NJ, Jakes RW, Rennie KL, Jo M, Hennings S, Day NE. Validity and repeatability of the EPIC-Norfolk Physical Activity Questionnaire. Int J Epidemiol. 2002;1:168-74

61. Knapp M, Beecham J. Reduced list costings: examination of an informed short cut in mental health research. Health Econ. 1993;4:313-22.

62. Diefenbach MA, Weinstein ND, O'Reilly J. Scales for assessing perceptions of health hazard susceptibility. Health Educ Res. 1983;8:181-92.

63. Kroenke K, Spitzer RL, Williams JB. The Patient Health Questionnaire-2: Validity of a Two-Item Depression Screener. Med Care. 2003;41:1284-94.

64. Watson M, Lloyd S, Davidson J, Meyer L, Ebbs S, Murday V. The impact of genetic counselling on risk perception and mental health in women with a family history of breast cancer. Br J Cancer. 1999;79:868-74.

65. Schwartz LM, Woloshin S, Black WC, Welch HG. The role of numeracy in understanding the benefit of screening mammography. Ann Intern Med. 1997;127:96-972

66. Ware JE, Kosinski M, Keller SK. SF- $36^{\circ}$ Physical and Mental Health Summary Scales: A User's Manual. Boston, MA: The Health Institute; 1994.

67. Ajzen I. Constructing a TpB Questionnaire: Conceptual and Methodological Considerations. 2002. http://www.uni-bielefeld.de/ikg/zick/ ajzen\%20construction\%20a\%20tpb\%20questionnaire.pdf. Accessed 25 April 2014.

68. Broadbent E, Petrie KJ, Main J, Weinman J. The Brief Illness Perception Questionnaire. J Psychosom Res. 2006;60(6):631-7.

69. Sanderson SC, Persky S, Michie S. Psychological and behavioral responses to genetic test results indicating increased risk of obesity: Does the causal pathway from gene to obesity matter? Public Health Genomics. 2010;13(1):34-47.

70. Zimet G, Dahlem N, Zimet S, Farley G. The Multidimensional Scale of Perceived Social Support. J Pers Assess. 1988;52:30-41.

71. Peretti-Watel P, L'Haridon O, Seror V. Time preferences, socioeconomic status and smokers' behaviour, attitudes and risk awareness. Eur J Public Health. 2013;23(5):783-8.

72. Lundberg O, Nyström Peck M. A simplified way of measuring sense of coherence: experiences from a population survey in Sweden. Eur J Public Health. 1995:5:56-9.

\section{Submit your next manuscript to BioMed Central and take full advantage of:}

- Convenient online submission

- Thorough peer review

- No space constraints or color figure charges

- Immediate publication on acceptance

- Inclusion in PubMed, CAS, Scopus and Google Scholar

- Research which is freely available for redistribution

Submit your manuscript at www.biomedcentral.com/submit 